\title{
Uma Arquitetura de Aplicação Gamificada para o Monitoramento e Tratamento da Doença Renal Crônica
}

\author{
Carlos Antonio Fernandes da Silva ${ }^{1}$, Alvaro Sobrinho ${ }^{1,2}$, Leandro Dias da Silva ${ }^{1}$ \\ ${ }^{1}$ Instituto de Informática - Universidade Federal de Alagoas (UFAL) \\ Caixa Postal 57500-000 - Maceió - AL - Brasil \\ ${ }^{2}$ Universidade Federal do Agreste de Pernambuco \\ Av. Bom Pastor, Garanhuns - PE - Brasil \\ \{cafs, leandrodias\}@ic.ufal.br, alvaro.alvares86@gmail.com
}

\begin{abstract}
Some computational applications focused on supporting the therapeutic treatment of Chronic Kidney Disease (CKD) are available in the market. However, up to this date, no software architecture has been found in the literature considering the various stages that CKD patients may present, seeking to change the lifestyle of patients by carrying out incentives and activities associated with the recommendations of nephrologists. Gamification is a relevant tool for providing game mechanisms to encourage patients to adhere to CKD treatment, providing challenges and rewards in the real world, similar to a digital game. This work presents a gamified application architecture with a focus on monitoring and treating CKD at different stages.
\end{abstract}

Resumo. Algumas aplicações computacionais com foco no apoio ao tratamento terapêutico da Doença Renal Crônica (DRC) são encontradas no mercado. Porém, até o presente momento, não foi constatada na literatura nenhuma arquitetura de software considerando os diversos estágios que o portador da DRC pode apresentar, buscando a mudança de estilo de vida de pacientes pela realização de incentivos e atividades associadas às recomendações de nefrologistas. A gamificação é uma ferramenta útil para a disponibilização de mecanismos dos jogos para incentivar a adesão de pacientes ao tratamento de DRC, proporcionando desafios e recompensas no mundo real, de maneira similar a um jogo digital. Neste trabalho é apresentada uma arquitetura de aplicação gamificada com foco no monitoramento e tratamento da DRC em diferentes estágios.

\section{Introdução}

A gamificação está relacionada com o uso de mecânicas dos jogos para gerar empenho e envolvimento por parte do usuário, fazendo com que a realização de tarefas se torne mais atrativa [Deterding et al. 2011]. A utilização da gamificação está atrelada a experimentação do usuário de um produto, através da criação de um ambiente que possibilite a sensação de bem-estar, permita o desenvolvimento de talentos e habilidades e estejam associadas premiações e recompensas virtuais ou físicas [Levey et al. 2005].

Aplicações de software relacionadas com os cuidados com a saúde e para a alteração do comportamento de usuários com relação à adesão ao tratamento de uma 
doença específica são exemplos de como a gamificação vem ganhando espaço no cenário atual.

$\mathrm{Na}$ presente pesquisa, o foco está no projeto de aplicações gamificadas para o monitoramento e tratamento de pacientes com doenças crônicas. Mais especificamente, $\mathrm{o}$ monitoramento e tratamento da Doença Renal Crônica (DRC).

Em algumas pesquisas, são implementados mecanismos de gamificação para pessoas com doenças crônicas. Por exemplo, no trabalho de Paim e Barbosa [Paim and Victoria Barbosa 2016] é apresento o Octopus, um modelo de gamificação para assistência no cuidado onipresente de doenças crônicas. Este busca incentivar o uso de recursos sensíveis ao contexto através da promoção de alteração no comportamento do usuário. Já na publicação de Berndt [Alexandre 2017], o objetivo foi identificar os elementos de gamificação e como utilizá-los no desenvolvimento de aplicações móveis de suporte a sessões terapêuticas de reminiscência ministradas a pacientes diagnosticados com doença de Alzheimer.

Neste trabalho, foram utilizados os conceitos definidos pelo método Attribute Driven Design (ADD) para especificar a arquitetura [Bachmann and Bass 2001]. Este se baseia na definição de atributos de qualidades que desejam ser obtidos, realizando, posteriormente, a decomposição da arquitetura até um nível de implementação. Os atributos de qualidade desejados neste trabalho são o atributo de modificabilidade e o atributo de escalabilidade [Bachmann and Bass 2001]. Deseja-se projetar uma arquitetura considerando a possibilidade de adição de novos módulos ou a alteração de algum módulo existente, de tal forma que impactos negativos nos artefatos já existentes sejam os menores possíveis. A escolha por esses atributos de qualidade se dá pelo fato da DRC ser divida em diferentes estágios (1, 2, 3A, 3B, 4 e 5), além da necessidade de um tratamento personalizado para cada estágio apresentado.

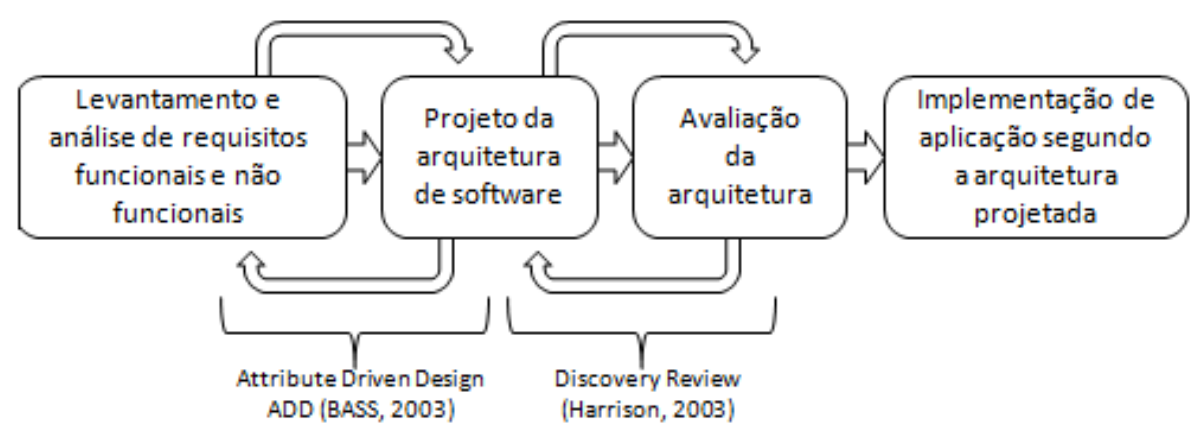

Figura 1. Esquema do processo de desenvolvimento usado para o projeto da arquitetura.

Para melhorar a confiança no projeto arquitetônico, uma abordagem baseada em cenários foi aplicada para avaliar a arquitetura proposta, entrevistando profissionais de saúde e um desenvolvedor de software. Um protótipo de aplicativo da web também foi desenvolvido como cenário de uso da arquitetura proposta. Assim, na etapa de avaliação da arquitetura, o padrão discovery review [Bachmann and Bass 2001] foi utilizado, conforme ilustrado na Fig. 1. As características da avaliação incluem pouca documentação, apresentação da arquitetura a ser projetada, partes interessadas e a coleta de feedback e 
indicações para melhorias, coletando feedback para realizar melhorias para gerar a arquitetura final.

\section{Caracterização do problema}

O portador da DRC, ao ser diagnosticado com a doença, precisa aderir a um estilo de vida diferente. Modificações na alimentação, adesão à prática de exercícios físicos e o uso de medicação são alguns exemplos de alterações na rotina do paciente.

A classificação nos estágios 1, 2, 3a, 3b, 4 e 5 indica que, para cada estágio da doença, existe um tratamento indicado [Levey et al. 2005]. Assim, baseando-se na terapia com o uso de uma aplicação de software para dar suporte ao tratamento, seriam necessários diferentes módulos de software para cada estágio da doença.

Em uma abordagem tradicional de projeto arquitetural, seriam necessários diferentes projetos para diferentes aplicações, aumentando custos e tempo de desenvolvimento. Assim, é desenvolvido um único projeto arquitetural que suporte as diferentes aplicações para os estagios existentes na DRC, incorporando características, como, por exemplo, reusabilidade de módulos, modificabilidade e escalabilidade funcional.

Desta forma, com este trabalho busca-se responder a questão de como disponibilizar uma arquitetura, dividida em módulos, que faz uso de gamificação, para motivar a alteração do comportamento, e se apliquem ao suporte terapêutico dos pacientes diagnosticados com DRC?

\section{Motivação da pesquisa}

A intervenção para DRC deve ser constante, uma vez que não possui cura. Portanto, é necessário que o paciente tenha ciência da sua natureza clínica. A obtenção de informações sobre as características da doença, como, por exemplo, os tipos de tratamento, instruções sobre a forma correta de utilização de equipamentos de hemodiálise ou diálise peritoneal e as indicações nutricionais recomendadas, são mecanismos que colaboram para uma melhor qualidade de vida do paciente [Levey et al. 2005].

O paciente portador da doença necessita de diferentes tratamentos e abordagens médicas considerando o estágio e nível de risco apresentado. Uma arquitetura de componentes pode permitir o desenvolvimento de novas aplicações baseadas no quadro de saúde atual do paciente.

Neste estudo é proposta uma arquitetura de aplicação gamificada para o tratamento e monitoramento da DRC. Neste contexto, a arquitetura proposta precisa da inserção de alguns dados e implementações específicas para o seu correto funcionamento. O cuidador, especialista da área da saúde, normalmente um médico, através de consulta médica, descreve o estágio atual da DRC do portador da doença. Em um cenário de uso, estes dados são inseridos em uma instância específica da arquitetura pelo ator desenvolvedor para gerar uma aplicação gamificada com foco no estágio da DRC identificado

\section{Objetivos e contribuições do trabalho}

O objetivo com este trabalho é auxiliar na elaboração de aplicações gamificadas de suporte a terapia e o automonitoramento da DRC, motivando os pacientes a efetuar as atividades terapêuticas. Para alcançar este objetivo principal, os seguintes objetivos específicos foram realizados: 
- Elicitar e especificar requisitos para aplicações com foco na DRC;

- Identificação de componentes arquiteturais de aplicações gamificadas para o monitoramento e tratamento de pacientes com DRC;

- Projetar e avaliar a arquitetura de software;

- Implementar um protótipo de aplicação, voltado para o estágio 1 da DRC, como cenário de uso e de avaliação da arquitetura proposta.

As principais contribuições obtidas com a realização deste trabalho são destacadas a seguir:

- Projeto de uma arquitetura de aplicações gamificadas para o monitoramento e tratamento da DRC, com a disponibilização de artefatos na forma de componentes de software agrupados em módulos.

- Disponibilização de um protótipo de aplicação desenvolvido com base na arquitetura proposta, voltado para o estágio 1 da DRC.

\section{Arquitetura proposta}

Para o projeto da arquitetura é realizado inicialmente o levantamento dos requisitos funcionais e não funcionais que deveram compor as aplicações geradas. Os requisitos funcionais, elicitados por meio de casos de uso para representar a interação entre o desenvolvedor, o usuário e a arquitetura proposta são mostrados na Fig. 2.

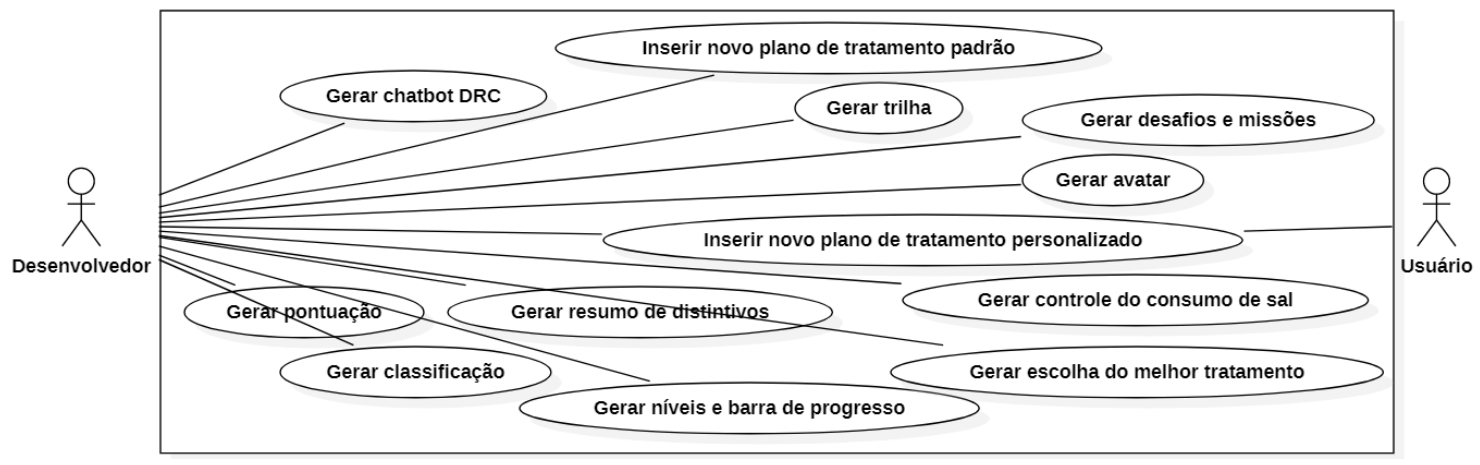

Figura 2. Diagrama de caso de uso.

Os requisitos não funcionais estão relacionados com os atributos de qualidade modificabilidade e escalabilidade funcional, e são utilizados para contemplar estes requisitos, seguindo o método ADD [Bachmann and Bass 2001].

O projeto da arquitetura é constituído por sua documentação. Nesta seção é apresentado um exemplo de visão arquitetural e seus elementos, documentados utilizando UML. Cada elemento arquitetural é constituído por um nome, a definição das suas atribuições, a interação com outros elementos e suas interfaces públicas.

A arquitetura proposta foi dividida em três módulos principais, cada um destes sendo formado por submódulos que realizam atividades fundamentais do sistema, conforme a Fig. 3. A decomposição em módulos foi realizada baseada no método ADD, seguindo um processo de decomposição onde, em cada estágio, táticas e padrões arquiteturais são selecionados para atender a um grupo de cenários de atributos de qualidade. 

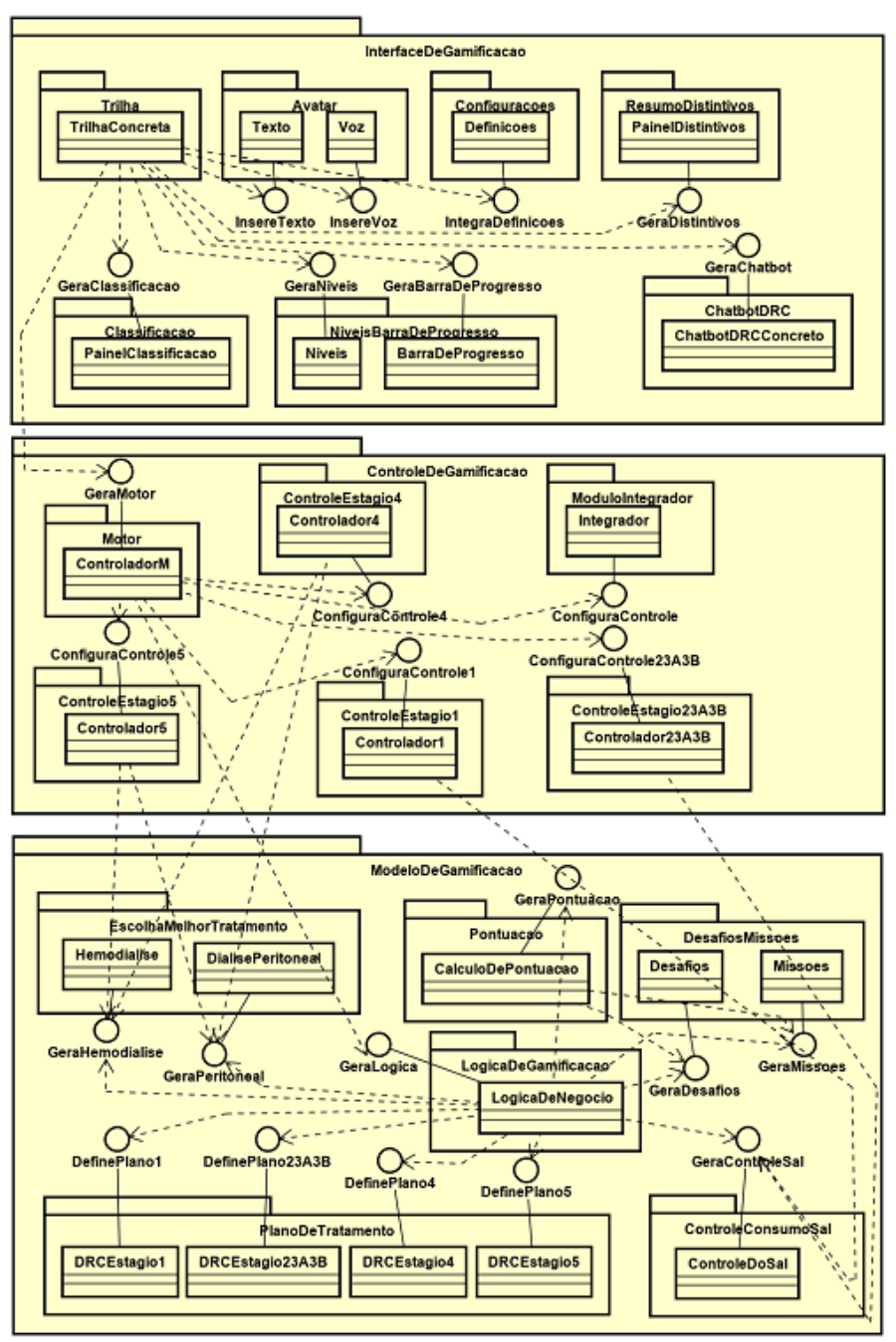

Figura 3. Diagrama de pacotes com classes e suas interfaces

\section{Avaliação da arquitetura}

A avaliação de uma arquitetura se faz necessário principalmente por ela ser a base de todo o sistema. Uma avaliação representa uma investigação da organização do sistema quanto a sua qualidade. A arquitetura proposta é voltada para interação com desenvolvedores, visando facilitar a tarefa de geração de aplicações e tornar a alteração do sistema e a personalização das aplicações facilitada, através da geração de diferentes aplicações de acordo com os módulos selecionados.

Neste trabalho foi implementado um protótipo de aplicação gamificada para o monitoramento e tratamento da DRC e identificados possíveis alternativas de implementação dos outros elementos da arquitetura. Vale ressaltar que a implementação deste protótipo é apenas uma das alternativas de implementação deste componente. Na Fig. 4 é apresentada uma amostra das interfaces graficas de usuário do protótipo desenvolvido.

Uma vez gerado o protótipo da aplicação, uma avaliação foi conduzida com os mesmos envolvidos no projeto da arquitetura (um médico, um desenvolvedor e dois enfermeiros). O objetivo é realizar uma análise técnica do que foi inserido no protótipo de aplicação, verificando se o protótipo apresenta um nível de satisfação, podendo, em um 

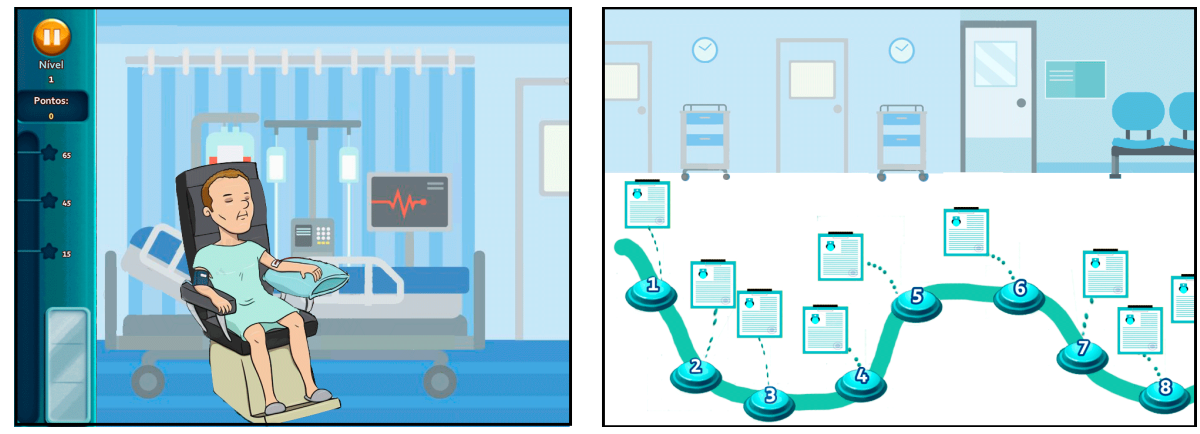

Figura 4. Interfaces gráficas de usuário para níveis, barra de progresso e trilha de atividades.

momento futuro, auxiliar o portador da DRC.

\section{Resultados obtidos e discussão}

A arquitetura foi definida para apoiar o desenvolvimento de aplicações, por desenvolvedores de software, com a colaboração de especialistas da área da saúde, como médicos e enfermeiros. Isto é relevante, por exemplo, para fornecer dados sobre o estágio da DRC no momento da consulta médica e sobre um quadro clínico geral do portador da doença.

Existe a possibilidade de continuidade deste trabalho para ampliação e consolidação. Por exemplo, as seguintes propostas podem ser destacadas: (1) utilizar as aplicações com os pacientes com DRC para a avaliação da efetividade da tecnologia proposta, e (2) inserir novos módulos na arquitetura, de acordo com a evolução da tecnologia ou a consideração de técnicas de tratamento específicas para cada estágio da DRC.

\section{Referências}

Alexandre, B. (2017). Uma arquitetura para desenvolvimento de aplicações gamificadas para suporte ao paciente com alzheimer. Master's thesis. Instituto de Informática - INF (RG).

Bachmann, F. and Bass, L. (2001). Introduction to the attribute driven design method. In Proceedings of the 23rd International Conference on Software Engineering, ICSE '01, pages 745-746, Washington, DC, USA. IEEE Computer Society.

Deterding, S., Dixon, D., Khaled, R., and Nacke, L. (2011). From game design elements to gamefulness: Defining "gamification". In Proceedings of the 15th International Academic MindTrek Conference: Envisioning Future Media Environments, MindTrek '11, pages 9-15, New York, NY, USA. ACM.

Levey, A. S., Eckardt, K.-U., Tsukamoto, Y., Levin, A., Coresh, J., Rossert, J., Zeeuw, D. D., Hostetter, T. H., Lameire, N., and Eknoyan, G. (2005). Definition and classification of chronic kidney disease: A position statement from kidney disease: Improving global outcomes (kdigo). Kidney International, 67(6):2089 - 2100.

Paim, C. A. and Victoria Barbosa, J. L. (2016). Octopus: A gamification model to aid in ubiquitous care of chronic diseases. IEEE Latin America Transactions, 14(4):19481958. 